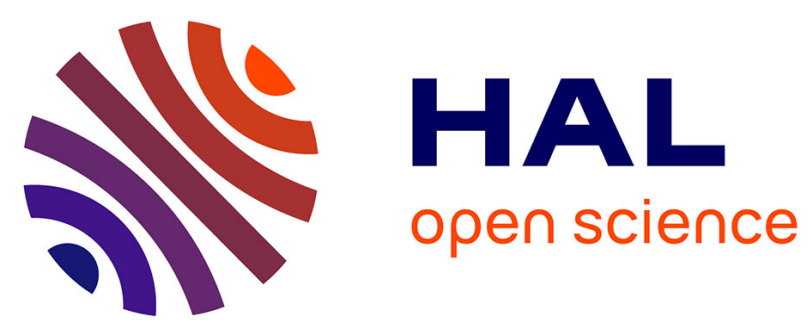

\title{
Soundscapes reveal disturbance impacts: biophonic response to wildfire in the Sonoran Desert Sky Islands
}

Amandine Gasc, Benjamin Gottesman, Dante Francomano, Jinha Jung, Mark Durham, Jason Mateljak, Bryan Pijanowski

\section{- To cite this version:}

Amandine Gasc, Benjamin Gottesman, Dante Francomano, Jinha Jung, Mark Durham, et al.. Soundscapes reveal disturbance impacts: biophonic response to wildfire in the Sonoran Desert Sky Islands. Landscape Ecology, 2018, 33 (8), 10.1007/s10980-018-0675-3 . hal-01837168

\section{HAL Id: hal-01837168 \\ https://hal.science/hal-01837168}

Submitted on 27 Mar 2019

HAL is a multi-disciplinary open access archive for the deposit and dissemination of scientific research documents, whether they are published or not. The documents may come from teaching and research institutions in France or abroad, or from public or private research centers.
L'archive ouverte pluridisciplinaire HAL, est destinée au dépôt et à la diffusion de documents scientifiques de niveau recherche, publiés ou non, émanant des établissements d'enseignement et de recherche français ou étrangers, des laboratoires publics ou privés. 


\title{
Soundscapes reveal disturbance impacts: biophonic response to wildfire in the Sonoran Desert Sky Islands
}

\author{
Amandine Gasc - Benjamin L. Gottesman - Dante Francomano • \\ Jinha Jung • Mark Durham · Jason Mateljak • Bryan C. Pijanowski
}

\begin{abstract}
Context While remote sensing imagery is effective for quantifying land cover changes across large areas, its utility for directly assessing the response of animals to disturbance is limited. Soundscapes approachesthe recording and analysis of sounds in a landscapecould address this shortcoming.

Objectives In 2011, a massive wildfire named "the Horseshoe 2 Burn" occurred in the Chiricahua National Monument, Arizona, USA. We evaluated
\end{abstract}

the impact of this wildfire on acoustic activity of animal communities.

Methods In 2013, soundscape recordings were collected over 9 months in 12 burned and 12 non-burned sites in four ecological systems. The seasonal and diel biological acoustic activity were described using the "Bioacoustic Index", a detailed aural analysis of sound sources, and a new tool called "Sonic Timelapse Builder" (STLB).

Results Seasonal biophony phenology showed a diurnal peak in June and a nocturnal peak in October in all ecological systems. On June mornings, acoustic activity was lower at burned than at non-burned sites in three of four ecological systems, due to a decreased

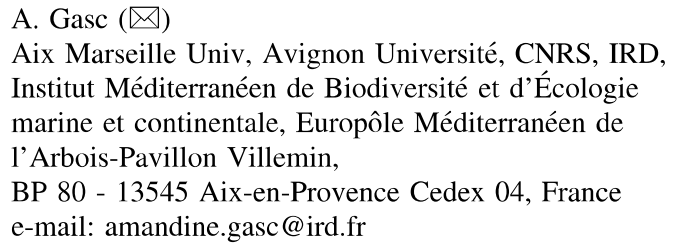
marine et continentale, Europôle Méditerranéen de l'Arbois-Pavillon Villemin, BP 80 - 13545 Aix-en-Provence Cedex 04, France e-mail: amandine.gasc@ird.fr

J. Jung

School of Engineering and Computer Science, Texas A\&M University, Corpus Christi, 6300 Ocean Drive, Corpus Christi, TX 78412-5797, USA

\section{Durham}

School of Media, Ravensbourne, London, 6 Penrose Way, Greenwich Peninsula, London SE10 0EW, UK

\section{J. Mateljak}

Lassen Volcanic National Park, 38050 State HWY 36, Mineral, CA 96063, USA 
abundance of cicadas directly impacted by the death of trees. Aural analyses revealed that $55 \%$ of recordings from non-burned sites contained insect sounds compared to $18 \%$ from burned sites. On October nights, orthopteran activity was more prevalent at some burned sites, possibly due to post-fire emergence of herbaceous.

Conclusions Soundscape approaches can help address long-term conservation issues involving the responses of animal communities to wildfire. Acoustic methods can serve as a valuable complement to remote sensing for disturbance-based landscape management.

Keywords Soundscape - Disturbance - Wildfire · Conservation biology $\cdot$ Remote sensing $\cdot$ Sonic timelapse

\section{Introduction}

A disturbance is defined as, "any relatively discrete event that disrupts the structure of an ecosystem, community, or population, and changes resource availability or the physical environment" (White and Pickett 1985). While in some cases disturbances can negatively affect the functioning of ecosystems and their resilience, many disturbances such as wildfire have beneficial effects on ecosystem dynamics (Turner et al. 1993; Turner 2010). Despite its destructive potential, wildfire plays essential roles in many ecosystems; it shapes forest structure (Heinselman 1981) and maintains grassland areas (Bond et al. 2005). Beginning in the 1960s after a half-century of wildfire suppression in the United States, fire has come to be recognized as an important tool for managing various ecosystems (Vankat 1977; Swetnam 1993; Duncan and Schmalzer 2004). The determination of appropriate fire management policy remains an active area of research, especially in a changing global environment where human activity and population growth are modifying climate conditions, fuel resources, and ignition frequency-the three primary drivers of wildfire (Dale et al. 2001; Guyette et al. 2002; Syphard et al. 2007; Turner 2010; Stephens et al. 2013). These changing conditions have heightened concerns regarding fire management strategies for natural areas (Halpin 1997; Hannah et al. 2007).

To evaluate the effectiveness of a fire management approach, researchers must monitor and analyze post- fire response in a given ecological system (Crotteau et al. 2013). To date, areas subject to such consideration are mainly monitored and managed at large spatial scales $\left(\mathrm{km}^{2}\right)$. Methods based on remote sensing imagery are extremely useful to survey wildfire impacts and ecosystem responses on such scales (Turner et al. 1994; Viedma et al. 1997; Röder et al. 2008). While these methods produce excellent results when assessing vegetation, they are limited in their ability to investigate animal dynamics. A review of fire impacts in the Mediterranean Basin explains why most post-wildfire monitoring surveys focus on vegetation, and why data on vegetation and animal communities rarely get integrated in a single largescale study: "Fauna changes due to fire are also omitted as they may require a very different approach" (Pausas et al. 2008). Indeed, large-scale disturbances currently require manual field collection as the primary method to directly assess the response and recovery of animal taxa, which cannot be implemented at the same resolution as remote sensing.

To complement satellite and aerial-based remote sensing methodologies, we demonstrate here that the use of soundscape analysis as a remote sensing method of high temporal resolution is able to detect changes in the composition and dynamics of animal communities following a wildfire disturbance. Soundscapes-defined here as the collections of sounds detected at given locations and time periods-are now considered a resource, a medium through which animals can communicate and extract information about their biotic and abiotic surroundings (Dumyahn and Pijanowski 2011; Pijanowski et al. 2011a, b; Schafer 1977). An entire research domain is growing around the idea that soundscape characteristics can reflect the ecological status and changes of a given location. Technical advances in automatic acoustic sensors (Acevedo and Villanueva-Rivera 2006), along with the development of acoustic diversity indices (Sueur et al. 2014), long-term soundscape visualization (Gage and Axel 2014; Towsey et al. 2014a), and acoustic event detection (Farina et al. 2018) have provided scientists with a new perspective for monitoring ecosystems at high resolution and large extent in both temporal and spatial domains. While numerous studies suggest that soundscape features can be used as indicators of biodiversity (Sueur et al. 2008b; Pieretti et al. 2011; Gasc et al. 2013a, b, 2015; Towsey et al. $2014 b$ ), only a few investigate the use of soundscape 
dynamics to evaluate the response of animal communities to disturbances (e.g. Joo et al. 2011; Deichmann et al. 2017; Lee et al. 2017).

The Chiricahua National Monument (CNM) is situated in southern Arizona at the eastern edge of the Sonoran Desert, where wildfire is a common disturbance. The CNM is part of the Madrean Archipelago, a series of "sky islands", or mountains that are isolated from one another by expanses of hot desert, driving microevolution and speciation on these mountains (Warshall 1995). The CNM alone is a site of remarkable biodiversity, harboring over 1200 species of plants and animals. Among these species, 1187 are documented as present, 25 others are listed as potential inhabitants, and 1117 are considered native (Powell et al. 2009). A dendrochronology study conducted in the CNM by Swetnam et al. (1992) concluded that mean fire intervals (MFI) between 1620 and 1890 varied between 3.9 years for fire scarring on any tree and 13.2 years for scarring on $>25 \%$ of trees, with fires occurring primarily in early summer. In May 2011, a campfire accident ignited the 5th largest fire in Arizona history. "The Horseshoe 2 Burn", as it was named, passed through the park in June of that year and burned approximately $82 \%$ of the CNM (U.S. Geological Survey and the U.S. Forest Service 2013).

The aims of this paper are to (1) describe the acoustic dynamics associated with different ecological systems of the CNM and (2) evaluate the differences between animal acoustic communities (i.e. the soniferous portion of the animal community; see Gasc et al. 2013b; Lellouch et al. 2014; Farina and James 2016) in burned and non-burned sites 2 years after this wildfire. To accomplish our objectives, we collected soundscape recordings between March and November 2014 in 24 different locations in the CNM. We analyzed these recordings by measuring the acoustic diversity of biophony (the portion of soundscapes produced by the acoustic community) and by using a new descriptive approach called "Sonic Timelapse" (STL), which allows for aural and visual exploration of long-term recording collections. The results presented in this paper describe Sonoran Desert soundscapes and illustrate the impact of wildfire on an ecosystem using soundscape analyses. Part of the discussion will focus on the possibilities of using soundscape recordings to monitor disturbance response processes and investigate the success of policies and management practices.

\section{Methods}

Site selection process

As suggested by Peters et al. (2011), the system properties for initial and response states of areas disturbed by wildfire need to be defined. The initial state, defined as the system state before the disturbance event, is here represented by the non-burned sites. The response state, defined as the system state after the disturbance event, is here represented by the burned sites. A comparison between the soundscapes of the initial state of different ecological systems and the soundscapes of the corresponding response state would reveal the effects of the fire on the soundscapes of that area. Following definitions in USGS GAP land cover data (U.S. Geological Survey 2011) that classify land cover based on the categories of Comer et al. (2003) at a $30 \mathrm{~m}$ resolution over the entire continental US, four main ecological systems of the CNM were considered: (1) "Madrean encinal" (997 hectares or $21 \%$ of the CNM) is dominated by several species of oaks (Quercus spp.) and graminoids (including Bouteloua spp., Eragrostis intermedia, and Muhlenbergia emersleyi); (2) "Madrean pine-oak forest" (611 hectares or $13 \%$ of the CNM) is composed of a pine (Pinus spp.) and oak (Quercus spp.) mosaic; (3) "Madrean pinyon-juniper woodland" (1902 hectares or $39 \%$ of the CNM) is dominated by pinyons (Pinus spp. and usually $P$. discolor) and is typically monospecific or mixed with junipers (Juniperus spp.) and manzanitas (Arctostaphylos spp.); (4) "Mogollon chaparral" ( 845 hectares or $17 \%$ of the CNM) is characterized by short oaks (Quercus spp.) and fireadapted evergreen shrubs, typically with small leaves.

For each of the four ecological systems, we acoustically monitored sites in each of two extreme burn states that we refer to as "burned sites" and "nonburned sites". Burn states were classified using data produced by the U.S. Forest Service's Burned Area Reflectance Classification (BARC) system (U.S. Geological Survey and the U.S. Forest Service 2013). This system categorizes sites in four categories from "nonburned" to different burn severity levels: "low", "medium", and "high". For all ecological systems, the "burned" sites selected were all in areas where burn severity was classified as "high" except for Mongollon chaparral, for which we selected sites where burn was classified as "medium" because high 
burn severity sites were exceedingly difficult to access because of the rock formations of the CNM.

To ensure that the soundscape differences between states were mainly due to the disturbance effect and not additional sources of variability, we selected 3 replicate sites for each of the 2 burn states and each of the 4 ecological systems, and we selected sites within each system that were as similar as possible (see Online Resource 1 for more details), leading to a total of 24 sites (Fig. 1; Online Resource 2).

Collecting acoustic recordings

Stereo recordings were collected using 24 digital field recorders (Song Meter SM2+; Wildlife Acoustics 2014), each outfitted with two omnidirectional microphones with flat frequency responses from $20 \mathrm{~Hz}$ to $20 \mathrm{kHz}$ (SMX-II; Wildlife Acoustics 2014). These programmable and weatherproof recorders were oriented horizontally at a height of $1.5 \mathrm{~m}$. A 10-min recording was made at the beginning of each hour. The sampling rate was $44.1 \mathrm{kHz}$, the gain was $36 \mathrm{~dB}$, and the bit depth was 16 bits. Recordings were collected from March to November in 2014 at each of the 24 sites mentioned, producing a total of 49,981 recordings that were collected and deposited in the sound library of the Center for Global Soundscapes (Purdue University, Indiana, USA).

Subsampling using meteorological data

Acoustic events that are not linked to biological activity can complicate acoustic analysis. To avoid misinterpretation of results, we excluded files containing these events from the analyses. Meteorological data were collected at the CNM station maintained by the Western Regional Climate Center of the Desert Research Institute (Nevada, USA). Hourly averaged wind speed and hourly precipitation were calculated from the raw data available at www.raws.dri.edu/cgibin/rawMAIN.pl?azACHR. These meteorological data were used to limit the following acoustic analyses to a subsample of the collected recordings excluding rare files containing rain noise and files from time periods with a wind speed above $3 \mathrm{~m} \mathrm{~s}^{-1}$. Given the low human activity in the park, we operated under the
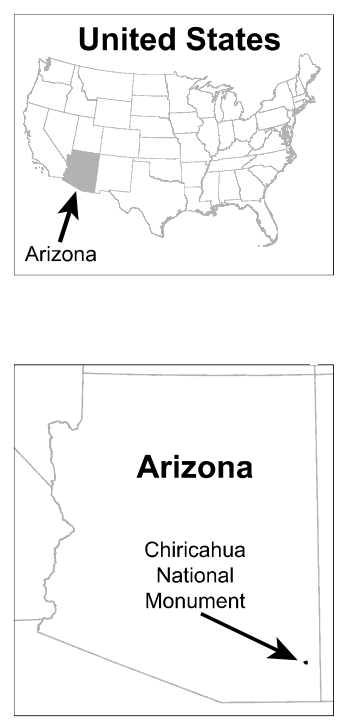
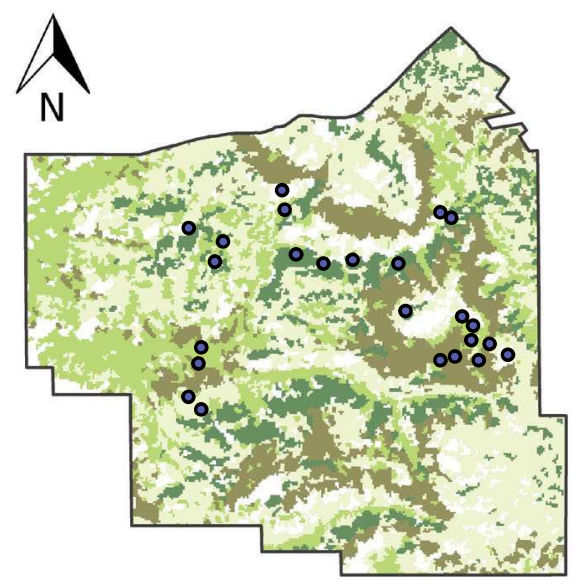

\section{Habitat types}

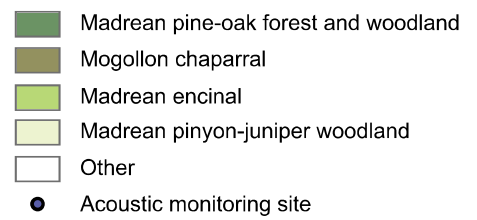



Burn states



Fig. 1 Maps of the Chiricahua National Monument. a Land cover of the four most abundant ecological systems: Madrean pinyon-juniper, Madrean encinal, Mogollon chaparral, and Madrean pine-oak forest. These data were collected before the wildfire of 2011 by USGS GAP. b Map of the Horseshoe 2 burn impact. On both maps, the dots represent the locations of the 24 acoustic monitoring sites 
assumption that the acoustic activity in these retained recordings was predominantly of non-human, biological origin. A total of 15,741 files $(31 \%)$ coincided with wind speeds above $3 \mathrm{~m} \mathrm{~s}^{-1}$, and 526 files (1\%) coincided with periods of rainfall, mostly during the monsoon period.

\section{Acoustic analysis}

We used three distinct and complementary techniques to analyze the acoustic data. Automatic calculation of an acoustic diversity index allowed for the consideration of a vast number of sound files, while detailed aural analysis enabled us to describe the contents of a subset of files in detail. Additionally, a new method, Sonic Timelapse, allowed for rapid aural appraisal of long-duration temporal dynamics. The synthesis of these three methods promoted a more complete understanding of these Sonoran Desert soundscapes.

\section{Acoustic diversity index}

Of the many acoustic diversity indices that were referenced in Sueur et al. (2014), we selected the Bioacoustic Index as appropriate for analysis of desert soundscapes. The Bioacoustic Index is defined by Boelman et al. (2007) as "a function of both the sound level and the number of frequency bands used by the avifauna", and by biological organisms more generally. Sound files were loaded into R ( Rx64 3.0.2; R Core Team, 2014) using "readWave" from the package $\{$ tuneR (Ligges et al. 2013). The index was then calculated using the function "bioacoustic_index" from the package \{soundecology (VillanuevaRivera and Pijanowski 2015). The index was only calculated on the left channel to reduce computation time. The "bioacoustic_index" function consists of five major steps. First, it performs a Fast Fourier Transform with a Hanning window set at 512 samples to obtain a spectrogram in three dimensions: time, frequency, and amplitude. The $\mathrm{dB}$ amplitude values of the spectrograms are relative values with a per-file maximum set to 0 . Second, it averages the spectrogram values over the temporal dimension (using the "meandB" function from the package \{seewave\}; Sueur et al. 2008a) to obtain a frequency spectrum of two-dimensions: frequency and amplitude. Third, it selects a user-specified portion of the averaged frequency spectrum (between 2 and $22 \mathrm{kHz}$ for this study). Fourth, it subtracts the minimum amplitude value from all the amplitude values. This step reduces the influence of background noise in the spectrum. Finally, it calculates the area under the curve of the frequency spectrum (the sum of the relative amplitudes for each frequency band). Because the Bioacoustic Index reduces the influence of background noise it allowed us to easily distinguish between recordings containing mostly background noise and those containing biological sounds (Boelman et al. 2007; Sueur et al. 2014; Gasc et al. 2015).

We then described the baseline non-burned biophonic patterns by examining the annual variation in the Bioacoustic Index for each of the four ecological systems at non-burned sites. Index values were averaged within each month and within daytime (0500-1800) and nighttime (1800-0500) periods. Based on this first analysis, we identified two peaks of biological activity: one in June during the daytime and a second in October during the nighttime. Further analyses focused on these peaks.

In order to evaluate the amount of between-site biophonic variability that could be explained by the burn state of sites, we computed Linear Mixed Models (LMMs) (Militino 2010). By focusing independently on the two periods of biophonic peak activity (June during daytime: 0500-1800 and October during nighttime: 1800-0500), we discarded seasonal variation from the subsequent analyses. We avoided temporal autocorrelation by calculating separate models for each hour independently ( $p$ values were corrected following the Bonferroni method; Holm 1979). In these models, the index value was considered as the dependent variable and was Box-Cox transformed to satisfy the normality assumption required for the model (Box and Cox 1964; Venables and Ripley 2002) using a lambda coefficient calculated with the function "boxcox" from the R package \{MASS \} (Venables and Ripley 2002). The burn state with two levels, "burned" and "non-burned", and the ecological system with four levels, "Madrean pinyonjuniper", "Madrean encinal", "Mogollon chapparal", and "Madrean pine-oak", were considered as independent categorical variables with fixed effects. Day of collection and site were added to the model as random effects (random intercept). We computed these models using the function "lmer" from the $\mathrm{R}$ package $\{1 \mathrm{me} 4\}$ (Bates et al. 2015). For each model, 
we tested for deviation of the residuals from linearity, homoscedasticity, and normality by visual inspection of the residuals, and we tested for collinearity between the fixed effects by calculating the Generalized Variation Inflation Factor using the function "vif" from the R package \{car\} (Fox and Weisberg 2011). The effect of burn state was evaluated using likelihood ratio tests that compared the full model against the model without the fixed effect of burn state (Bolker et al. 2009; type I error rate at 1\%).

\section{Aural descriptions}

To aid in our interpretation of significant differences in acoustic diversity index values observed between burned and non-burned areas, we developed a protocol for aural description of soundscape recordings. Amandine Gasc (AG) listened to the first min of each file and noted the percentage of time occupied by each of three sound categories (biophony, geophony, and anthrophony; Pijanowski et al. 2011b). Furthermore, within each category, AG made a more detailed subclassification (outlined in Table 1). This protocol was used to describe the soundscape recordings collected throughout the CNM ( $n=114$ recordings for each burn state) in June at 0900 , as that period of time was shown to be significant in the previously described statistical tests.

\section{Sonic Timelapse}

Recordings collected for the months of June and October were also analyzed using "Sonic Timelapse Builder" (STLB). Benjamin Gottesman and Mark Durham developed this program as a method for rapid evaluation of large soundscape collections using humans' aural senses. The audio files produced by this method, called "Sonic Timelapses" (STLs), are sonic summaries of vaster acoustic data that are brief but information rich. While STLs are usually produced using recordings from a single site, they can also incorporate recordings from multiple sites through a pre-processing averaging step (detailed below). For the present study, a total of 16 STLs were produced,

Table 1 Manual annotation after listening to each recording

\begin{tabular}{ll}
\hline Category & Rating \\
\hline Geophony & Total occurrence duration: $0,1-25,26-50,51-75$, or $76-100 \%$ \\
Wind & $0=$ absence, $1=$ light wind, $2=$ strong wind with movement of leaves, or $3=$ strong wind with clipping \\
Rain & $0=$ absence, $1=$ light rain with differentiable drops or $2=$ strong rain with non-differentiable drops \\
Water flow & $0=$ absence, $1=$ stream, or $2=$ rushing stream \\
Waves & $0=$ absence or $1=$ presence \\
Thunder & $0=$ absence or $1=$ presence \\
Biophony & total occurrence duration: $0,1-25,26-50,51-75$, or $76-100 \%$ \\
Bird & range of sound type numbers: $0,1-3,4-6,7-9$, or $>9$ \\
Insect & range of sound type numbers: $0,1-3,4-6,7-9$, or $>9$ \\
Mammal & range of sound type numbers: $0,1-3,4-6,7-9$, or $>9$ \\
Amphibian & range of sound type numbers: $0,1-3,4-6,7-9$, or $>9$ \\
Reptile & range of sound type numbers: $0,1-3,4-6,7-9$, or $>9$ \\
Anthrophony & total occurrence duration: $0,1-25,26-50,51-75$, or $76-100 \%$ \\
Talking & $0=$ absence or $1=$ presence \\
Walking & $0=$ absence or $1=$ presence \\
Plane & $0=$ absence or $1=$ presence \\
Car & $0=$ absence or $1=$ presence \\
Boat & $0=$ absence or $1=$ presence \\
Other motors & $0=$ absence or $1=$ presence \\
Gunshot & $0=$ absence or $1=$ presence \\
Alarm & $0=$ absence or $1=$ presence \\
\hline
\end{tabular}


with 1 for each ecological system $(n=4)$, burn state $(n=2)$, and month of interest $(n=2)$.

Data were first prepared by randomly selecting 5 recordings per hour for each site and each month, leading to a total of 5760 sound files (Fig. 2, Step A). Then, all recordings from the same hour, month, ecological system, and burn state were averaged (Fig. 2, Step B), reducing the number of files to 384 (see the two $\mathrm{R}$ scripts used for data preparation, "SLTDataPrep.r" and "STLDataPrepAverage.r", at https://github.com/agasc/Soundscape-analysis-with$\mathrm{R}$; these R scripts can be used on any Pumilio system libraries described by Villanueva-Rivera and Pijanowski (2012)). Averages were the means of waveforms (i.e. averages of vectors of the same length).
Finally, STLs were produced from these recordings using STLB with a slice length of $2000 \mathrm{~ms}$ for each file and a file overlap setting of 2 , which cross-fades between two files by delaying the start of the following file by $25 \%$ of the slice length (Fig. 2, Steps C and D). With this setting, four consecutive files play simultaneously, which reduces listening time by approximately $75 \%$. A $2 \mathrm{kHz}$ high pass filter was applied. This program was written in Max/MSP (code available at https:/github.rcac.purdue.edu/PijanowskiGroup/ Sonic-Time-lapse-Builder; see Online Resource 3 for more details about the program). STLs were examined aurally and visually through their corresponding spectrograms. The STL files used in the presented analyses and figures are presented in Online Resources
Fig. 2 Diagram of Sonic Timelapse computation. Grey rectangles, arrows, and sentences represent acoustic recordings, directions of transformation or computation, and descriptions of each computational step, respectively. The STL length is $13.7 \mathrm{~s}$ instead of $13.5 \mathrm{~s}$ due to Max/MSP program settings




6-22 in .mp2 format (this file format was necessary for inclusion as Online Resources; it imposes low-pass filtering around $15 \mathrm{kHz}$, but most content above that frequency was negligible in the context of this study). The amplitude of these original files is low, and we recommend some amplification when listening to them.

\section{Results}

Soundscape activity at the Chiricahua National Monument

Numerous geophonic events were recorded at the CNM, particularly during the annual monsoon period from July to September that brings wind, thunder, and heavy rain to the area (Fig. 3a). As for biophony, it exhibited a strong seasonality that peaked during short periods of time across the four ecological systems considered in this study (Fig. 3b). In daytime recordings, the Bioacoustic Index peaked in June, while in nighttime recordings it peaked in October.

\section{Daily patterns in different non-burned systems}

As expected, within June and October, daily biophonic patterns varied between non-burned ecological systems (unbroken line in Fig. 4a for June and Figure S1A in Online Resource 4 for October). In June, all four systems exhibited varying increases in acoustic activity between 0700 and 1000 . A second activity peak at 1600 was only observed for Madrean pinyon-juniper and Madrean pine-oak systems. Based on aural evaluation of the STLs, we suspect that these activity peaks could be associated with insect and especially cicada sounds that cover a wide band of frequencies during those time periods in non-burned sites. In October, index values were higher at night with a peak between 1900 and 2100 .

The impact of wildfire on biophony

We first considered the impact of wildfire on biophony through graphical observation of the value of the Bioacoustic Index in both burned and non-burned areas. These initial observations were supplemented by listening to STLs and observing their corresponding spectrograms (Fig. 4b). We then ran statistical tests and conducted detailed aural analyses on a subsample of files corresponding to significant test results.

For June, the daily variation of the Bioacoustic Index in non-burned areas was clearly different from its variation (or lack thereof) in corresponding burned areas for Madrean pinyon-juniper, Madrean encinal, and Mogollon chaparral, especially during the daytime in June (Fig. 4). In parallel, the STLs showed an absence of cicadas for Madrean pinyon-juniper and Mogollon chaparral and reduced cicada activity in Madrean encinal and Madrean pine-oak areas suggesting that (1) the Bioacoustic Index was influenced by the cicada sounds, and (2) the difference observed between burned and non-burned sites might be explained by the absence or reduced abundance of cicadas in burned sites. The difference between nonburned and burned curves for Madrean pine-oak sites did not allow for visual interpretation.

A difference between values of the Bioacoustic Index in non-burned and burned sites considering all ecological systems was confirmed with a significant difference at $0900\left(\chi^{2}(1)=37.917, P<0.001\right)$. For other times of day there were no significant differences, or the assumption of normality of the residual distribution was not satisfied and the results could not be used (see Online Resource 5 for detailed results of the likelihood tests and validation of assumptions).

The detailed aural sound descriptions performed at 0900 indicated that more files contained biological sounds and fewer files contained no audible sounds (i.e. recordings without geophony, biophony, or anthrophony) at non-burned sites compared to burned sites (Fig. 5). Focusing on biophony, the results of this aural description confirmed the following: (1) a higher proportion of recordings from non-burned sites contained insect sounds (55\%) than recordings from burned sites (18\%; Fig. 6b); (2) insect sounds occupied a greater percentage of time in recordings in which insect sounds were heard than bird sounds occupied in recordings in which bird sounds were heard (Fig. 6a). Aural detection of cicada sounds revealed that $26.4 \%$ of recordings from non-burned sites contained cicada sounds, while only $7.2 \%$ of the recordings from burned sites contained cicada sounds. Other insect sounds include those of Orthoptera.

For the month of October, the daily variations of the Bioacoustic Index were generally similar between burned and non-burned sites (see Online Resource 4). 
Fig. 3 Seasonal variation at the Chiricahua National Monument. a Variation of meteorological parameters, average value per day. The grey box represents the monsoon period. b Time series of the average value of the Bioacoustic Index per month for each ecological system for daytime (0500-1800) and nighttime (1800-0500). The grey box represents the peak of acoustic activity in the year

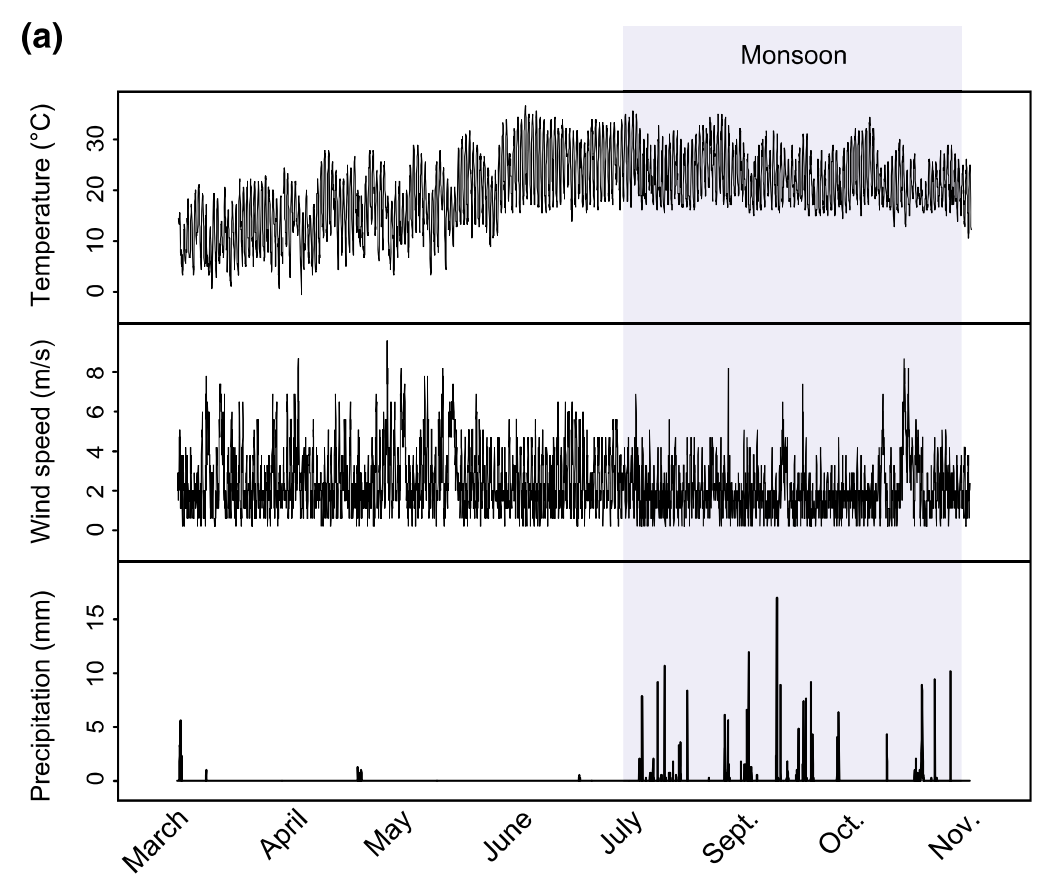

(b)

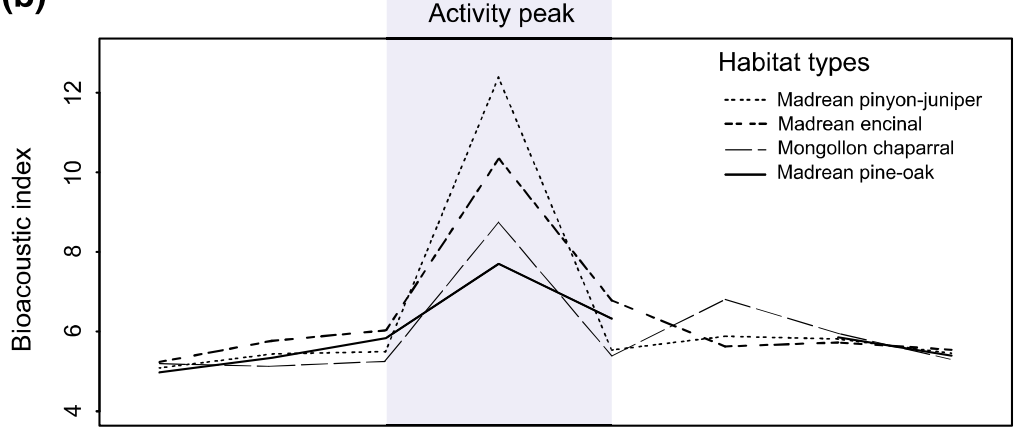

Daytime

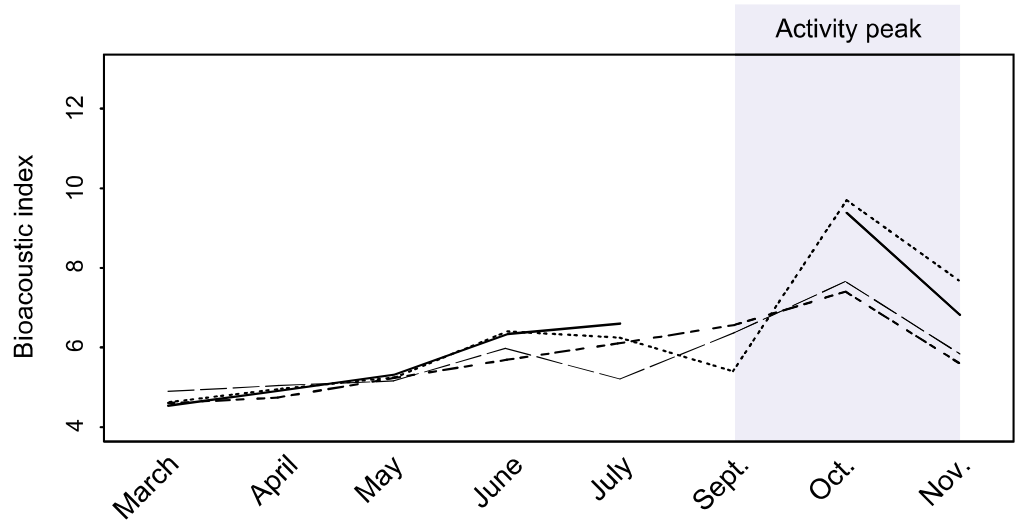

Nighttime
The associated statistical tests were not able to show any significant differences between Bioacoustic Index values from burned and non-burned sites (see Online
Resource 5 for details concerning model results and the validation of statistical assumptions). However, some differences in the Bioacoustic Index were visible 
(a)



Fig. 4 Diel acoustic variation for June in non-burned and burned sites. a Variation of the Bioacoustic Index for each of the four ecological systems. Each point represents the average and each bar represents the standard error associated with the index value. The number of sites used for averages varied due to missing data: 2 non-burned and 1 burned site for Madrean pinyon-juniper, 3 non-burned and 3 burned sites for Madrean

for Madrean encinal and Mogollon chaparral sites at night. Statistical tests were not possible for these specific sites because of a lack of replicates due to a large amount of missing data resulting from recorder failure due to rechargeable batteries that did not last as long as expected (see Online Resource 4), but aural evaluation of STLs revealed that in both Madrean encinal and Mogollon chaparral, the burned sites exhibited a more intense and diverse night-time soundscape than the non-burned sites, though differences in the Madrean encinal were more pronounced. Nighttime soundscapes in these two ecological systems contained two main frequency bands of cricket (Grillidae spp.) activity, from 2 to 3 and 3.5 to $4.5 \mathrm{kHz}$. The burned areas in Madrean encinal had (b)

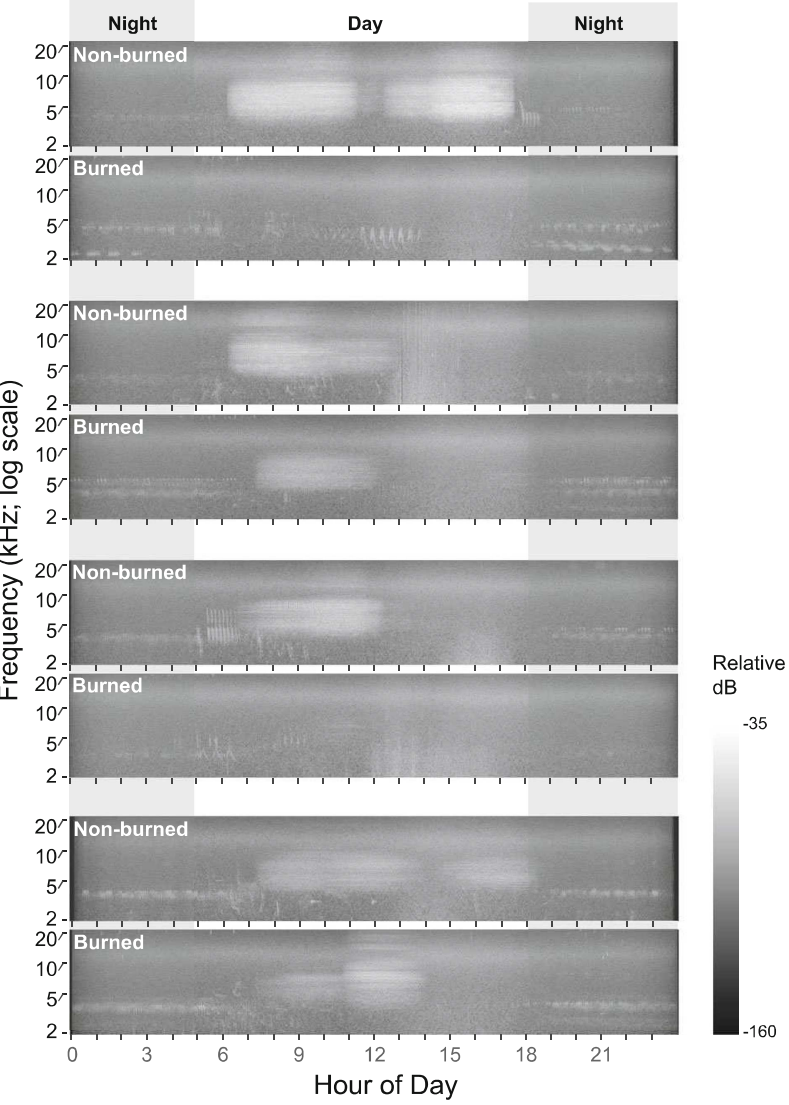

encinal, 2 non-burned and 2 burned sites for Mogollon chaparral, and 3 non-burned and 3 burned sites for Madrean pine-oak. b Spectrograms of the corresponding Sonic Timelapses (STLs) for non-burned and burned areas of each ecological system. Spectrograms were derived from $13.7 \mathrm{~s}$ STLs with a high pass filter set at $2 \mathrm{kHz}$

large amounts of these two sounds, while the nonburned area only had a faint presence of cricket sound from 3.5 to $4.5 \mathrm{kHz}$. In the Mogollon chaparral, both sound types were present, but the band from 2 to $3 \mathrm{kHz}$ was more intense in the burned area.

\section{Discussion}

Long-term desert soundscape recordings along with a new analytical methodology (STL) were used to determine how animals responded to wildfire in different ecological systems of the Madrean Archipelago sky islands in the Sonoran Desert. Two years after the Horseshoe 2 Burn the soundscape phenologies that 
Fig. 5 General composition of the recordings in terms of biophony, geophony, and anthrophony. The bar plots represent the number of recordings for each category from the 114 recordings collected from non-burned sites and the 114 recordings collected from burned sites. Venn diagrams complete this representation by accounting for recordings with multiple categories of sound sources

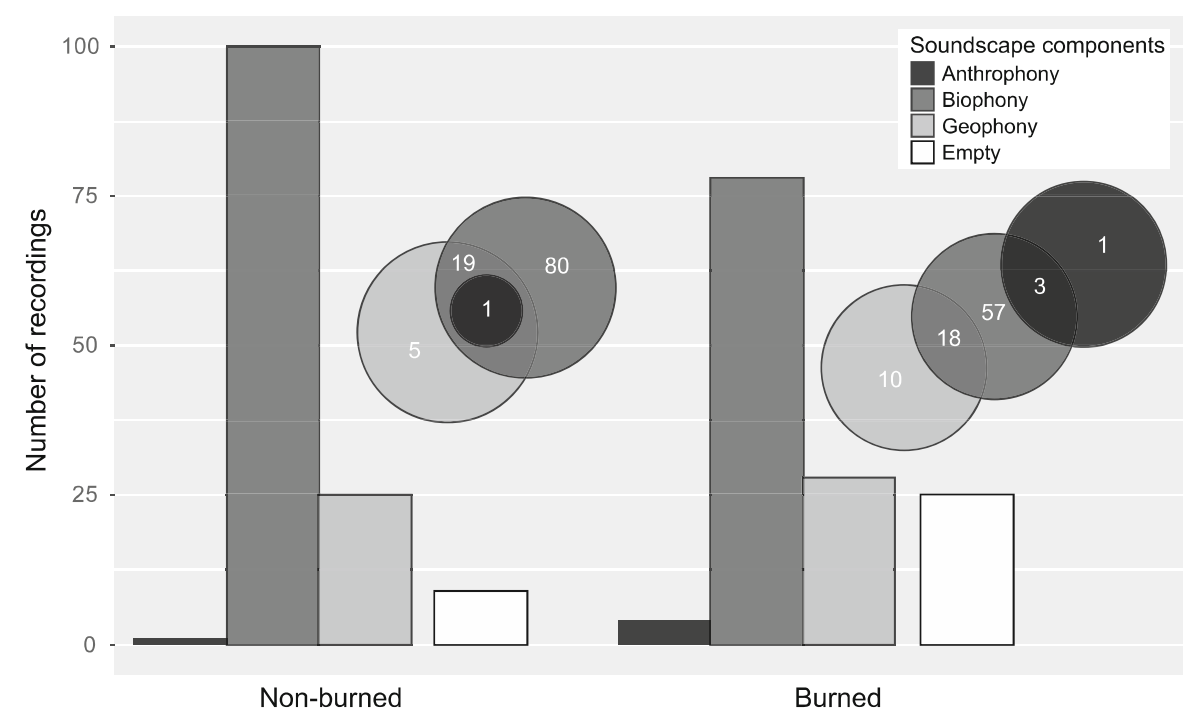

(a)

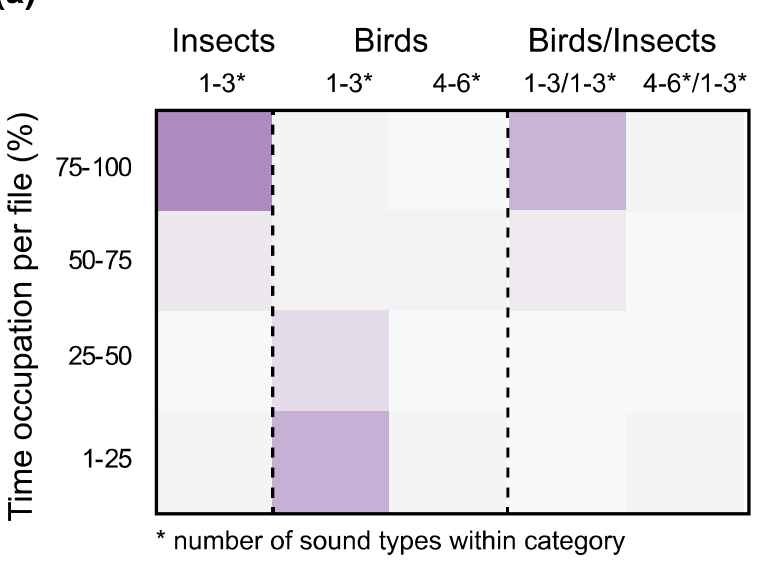

Burned



(b)

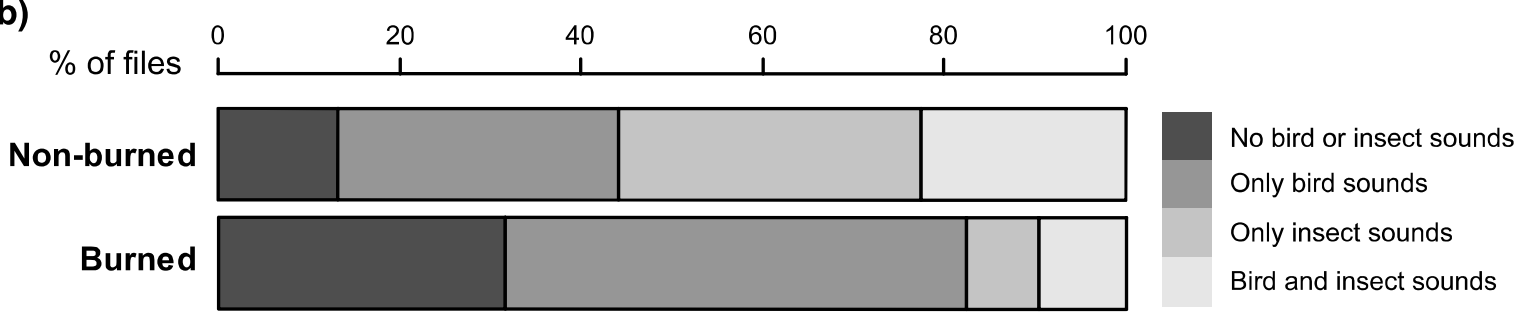

Fig. 6 Results of detailed biophonic composition analysis. a A 3-dimensional representation of the biological sounds identified in the recordings. The $\mathrm{x}$-axis represents the different classes of the soundscape composition (in terms of taxa and number of sound types), and the $y$-axis represents the time of occupation of

we documented allowed us to highlight higher numbers of biophonic events at non-burned sites mainly related to the higher number of insect sounds, lower these sounds in the recordings. The third dimension shown by the color gradient represents the percentage of recordings. b Percentage of files for different classes of soundscape composition for both non-burned and burned sites

acoustic activity from cicadas (in the morning in June) at burned sites, and also higher acoustic activity from crickets at the burned sites of Mogollon chaparral and 
Madrean encinal (at night in October). These findings must be considered as a snapshot of the response processes following the disturbance. However, soundscape approaches can certainly be implemented immediately after a disturbance (or even before anticipated disturbances) and applied over longer periods of time. Below, we discuss the implication of these results for both soundscape and fire/disturbance ecology.

\section{Natural soundscape phenologies}

The Sonoran Desert is far from silent (cf. Lomolino et al. 2015); rather, its soundscape is composed of sparse but diverse biological sounds along with geophonic events stemming from frequent wind and periodic monsoon rain. Inventories directed by the CNM have described numerous species producing sound: 91 species of mammals (about half of which are bats), 4 species of frogs and toads, 221 species of birds, and many species of insects (CNM Inventories accessed on 2015/08/09). From our aural observations of July and October recordings, bird and insect sounds are predominant in CNM soundscapes.

Beyond the richness of sounds, we would like to emphasize the importance of describing diel and seasonal acoustic phenologies in soundscape analysis. Diel and seasonal phenologies are important descriptors of soundscapes, reflecting ecological patterns. This standard measure of soundscapes can be applied in all ecosystems, both terrestrial and aquatic, and it is relevant across disturbance gradients (Gasc et al. 2016). Diel patterns of acoustic communities have been described by aurally detailing the acoustic activity of the species composing the community (Drewry and Rand 1983; Diwakar and Balakrishnan 2007; Luczkovich et al. 2008). Additionally, the scientific community has recently adopted more holistic description of diel soundscape variation (Pekin et al. 2012; Kuehne et al. 2013; Gage and Axel 2014; Fuller et al. 2015; Mullet et al. 2016; Farina et al. 2018) and seasonal soundscape variation (Krause et al. 2011; Pijanowski et al. 2011b; Farina and Pieretti 2014; Gage and Axel 2014) in different ecosystems.

To complement these existing phenologies, the present study of desert soundscapes shows clear diel and seasonal patterns with low acoustic activity for much of the year except for two high-activity periods attributed to bird and insect activity. In comparison with previous soundscape phenology descriptions, this phenology is similar to those of temperate terrestrial systems (Depraetere et al. 2012; Gage and Axel 2014), but it exhibits a higher amplitude difference between periods of low and high acoustic activity. The Sonoran Desert has extreme fluctuations of temperature and precipitation that have led to adaptive biological strategies, and biophonic patterns could simply reflect those strategies. The peaks in the phenology of the Sonoran Desert biophony, as measured by the Bioacoustic Index, are likely associated with insect emergence. Cicadas in this study area are likely responsible for the June daytime peak in biophonic activity, as they tend to emerge in early summer and are acoustically active during the daytime (Ellwood et al. 2012). In October, the ecological explanation of the biophony peak is less clear, but it may be a result of nighttime insects (e.g., Orthoptera) that are exploiting the food and shelter afforded by increased vegetation following the monsoon season rainfall (Watts et al. 2007; Méndez-Barroso et al. 2009).

Monitoring disturbance impacts and responses

Cicadas were likely the primary drivers of the June difference in soniferous animal activity between burned and non-burned sites. Forty-seven species of cicadas have been identified in Arizona (Sanborn and Phillips 2013), of which at least Oncometopia spp. and Neotibicen canicularis are present in the CNM (unpublished inventories conducted by the CNM; Hill et al. 2015). In addition, the primary soniferous cicada species on our recordings has been identified as Hadoa duryi, an annual cicada common in deciduous woodlands of the American Southwest. There are three hypotheses as to how wildfire might impact the presence and abundance of cicadas. First, it may deplete food resources for cicadas by degrading the quality of belowground root tissue and aboveground vegetation that are consumed by larvae and adults, respectively (Callaham et al. 2000). More specifically, the absence or reduced abundance of living trees in severely burned areas could explain the decreased abundance of soniferous adult male cicadas (Chiavacci et al. 2014; Fonseca 2014). Second, wildfire may limit the availability of oviposition sites aboveground for certain species of cicadas (Yeates and Lee 1997; Callaham et al. 2003). Finally, predation 
pressure from birds might increase due to the reduction in protective vegetation (Williams et al. 1993).

In October nighttime soundscapes of two ecological systems (Madrean encinal and Mogollon chaparral), burned sites had higher and more diverse biophonic activity than non-burned sites, due to crickets and other orthoptera. This difference could be explained by the increased availability of food and shelter for orthoptera linked to the increase of low vegetation (grasses and herbaceous plants) during the initial post-fire response phase (Engle et al. 2008; Röder et al. 2008).

Different animal taxa obviously respond differently to the environmental changes occurring after a fire. In burned sites 2 years after the Horseshoe 2 Burn, acoustic activity of orthoptera was higher and acoustic activity of cicadas was lower, corresponding to the early stage of vegetational succession in which low vegetation is present, but trees have not yet recovered (personal observation). Tracking changes in animal community diversity can enhance monitoring of the overall ecosystem disturbance response process. Soundscape approaches allow for the necessary long temporal extent and high temporal resolution to consider various taxa that exhibit distinct seasonal and diel dynamics. The present study should be replicated at the same sites in the future to determine the extent to which acoustic activity correlates with further phases of post-fire responses.

The conclusions reached in this study were limited by a paucity of replicates and frequent battery failure, which meant that most small differences in Bioacoustic Index values between burned and non-burned sites that were seemingly consistent across ecological systems did not yield statistically significant results. For future research projects using soundscape analyses to assess the impacts of fire or other disturbances in the landscape, we highly recommend using more replicates per treatment (more sites) to detect significant statistical results with smaller effect sizes. We also recommend the use of solar panels to improve recorder longevity, especially in remote sites with abundant sunlight. Additionally, the long-duration, broadband sounds of cicadas likely influenced the Bioacoustic Index towards very high values for recordings in which cicada sounds were present (as might be expected with other acoustic indices). The extreme difference in index values between recordings with and without cicada sounds may have masked subtler differences resulting from varying abundances or acoustic activity of other insects or birds, which is why we employed two additional complimentary techniques (STL and detailed aural analysis). We also recommend working with taxonomists who could help in the aural identification of other taxonomic groups (e.g., crickets and birds), and exploring the use of automated detection algorithms to seek targeted sounds (Ruse et al. 2016; Zhao et al. 2017).

\section{Implications for soundscape and fire/disturbance ecology}

Managers strive to control fire intensity and propagation speed to prevent uncontrollable burns and to promote fire regimes that support ecosystem resilience. In this regard, soundscape approaches are poised to offer valuable information at high spatial and temporal resolution concerning the responses of animal communities to fire disturbances. Thus, they complement extant remote sensing imagery that is used to monitor vegetation responses. Inspired by the development of remote sensing imagery, soundscape approaches are still being advanced through (1) remote data collection and measurement, (2) measurement calibration, and (3) automation of collection, measurement, and analysis. However, current soundscape analysis methods are already able to highlight differences in acoustic animal community composition between burned and non-burned sites, supporting the application of soundscape ecology approaches to fire ecology and the evaluation of fire management practices. Studies that begin soundscape recording before fires and extend many years beyond the fire date would provide even better reference data against which to compare future fire impact studies.

Soundscape analyses can be applied over large spatial and temporal extents, but such application generates big data issues. While manual analysis may be able to identify soundscape components with greater accuracy and nuance, it is almost impossible to implement on such scales due to the "1:1 relationship between the data and its analysis" (Truskinger et al. 2013). This bottleneck has deterred scientists from listening to their acoustic data. Instead, the automatic calculation of acoustic diversity indices is the primary method of acoustically assessing biodiversity and ecosystem health (Sueur et al. 2014; Towsey et al. 2014b). While reliance on these metrics 
is desirable for large-scale implementation of soundscape approaches, the interpretation of these metrics has been challenging for ecologists and can be biased by sounds that are not of interest (Fairbrass et al. 2017). In this context, aural analysis remains a valuable and sometimes necessary tool to link biological processes with the output of automated analyses. To describe soundscape composition and dynamics, we adopted a tripartite methodology that employed acoustic diversity indices, STLs, and detailed aural description. This mixed-method approach improved our interpretation of acoustic index results, and therefore, we recommend such approaches for analysis of long-term recordings.

While "long-term spectrograms"- -spectra sequentially combined into one long image - have been used, they only create a visual representation of recordings (Sankupellay et al. 2015). STLs offer a means to comprehensively screen large audio time series using aural and visual senses. STLs can aid in identifying acoustic events linked to anthropic activity, weather, and/or equipment failure that can bias automated analysis (Digby et al. 2013) and reveal long-term phenological trends. Considering the results of acoustic indices while listening to the corresponding STLs can contribute to an understanding of the drivers of soundscape patterns, and it can aid in the selection of subsamples for more detailed aural description of soundscape components. As more automated tools become incorporated into land management practices and conservation efforts, developing efficient systems to ground-truth automated analyses will be important to ensure proper interpretation of results.

Soundscape analysis presents an opportunity for more complete monitoring of response processes following disturbance events and for evaluating the impact of post-disturbance landscape management strategies. Coupled with remote sensing imagery and knowledge of species' life history, behavior, and sound production, soundscape ecology represents a powerful analytical approach to enhance our understanding of disturbance responses and facilitate adaptive landscape management.

Acknowledgements We would like to thank our field assistant Karen Krebbs as well as Maura Thoenes Buckley from the Chiricahua National Monument for their help in the protocol development and the collection of the data. Additionally, we would like to thank Matthew Harris and Marc Manceau for their help in the field and their work in the documentation of this study. We are grateful for the helpful and constructive comments of the two anonymous reviewers.

Funding This work was partially funded by the Wright Forestry Fund of the Department of Forestry and Natural Resources, the Purdue University Graduate School, National Science Foundation Research Coordination Networks (NSF RCN \#1114945), National Science Foundation Division of Information and Intelligent Systems (NSF IIS \#0705836), Purdue University's Center for the Environment, the United States Department of Education's Graduate Area of National Needs (GANN) Program, the McIntire-Stennis Cooperative Forestry Research Program of the U.S. Department of Agriculture, the College of Agriculture at Purdue University, and the Executive Vice President for Research and Engagement at Purdue University.

\section{Compliance with ethical standards}

Conflict of interest The authors declare that they have no conflicts of interest.

\section{References}

Acevedo MA, Villanueva-Rivera LJ (2006) Using automated digital recording systems as effective tools for the monitoring of birds and amphibians. Wildl Soc Bull 34:211-214

Bates D, Maechler M, Bolker B, Walker S (2015) Fitting linear mixed-effects models using lme4. J Stat Softw 67:1-48

Boelman NT, Asner GP, Hart PJ, Martin RE (2007) Multitrophic invasion resistance in Hawai' I : bioacoustics, field surveys, and airborne remote sensing. Ecol Appl 17:2137-2144

Bolker BM, Brooks ME, Clark CJ, Geange SW, Poulsen JR, Stevens MH, White JS (2009) Generalized linear mixed models: a practical guide for ecology and evolution. Trends Ecol Evol 24:127-135

Bond WJ, Woodward FI, Midgley GF (2005) The global distribution of ecosystems in a world without fire. New Phytol 165:525-538

Box GEP, Cox DR (1964) An analysis of transformations (with discussion). J R Stat Soc B 26:211-252

Callaham MA Jr, Blair JM, Todd TC, Kitchen DJ, Whiles MR (2003) Macroinvertebrates in North American tallgrass prairie soils: effects of fire, mowing, and fertilization on density and biomass. Soil Biol Biochem 35:1079-1093

Callaham MA Jr, Whiles MR, Meyer CK, Brock BL, Charlton RE (2000) Feeding ecology and emergence production of annual cicadas (Homoptera: Cicadidae) in tallgrass prairie. Oecologia 123:535-542

Chiavacci SJ, Bednarz JC, McKay T (2014) The emergence densities of annual cicadas (Hemiptera: Cicadidae) increase with sapling density and are greater near edges in a Bottomland Hardwood Forest. Environ Entomol 43:859-867

Comer P, Faber-Langendoen D, Evans R, Gawler S, Josse C, Kittel G, Menard S, Pyne M, Reid M, Schulz K, Snow K, 
Teague J (2003) Ecological systems of the United States: a working classification of U.S. terrestrial systems. NatureServe, Arlington, Virginia

Crotteau JS, Varner JM, Ritchie MW (2013) Post-fire regeneration across a fire severity gradient in the southern Cascades. For Ecol Manage 287:103-112

Dale VH, Joyce LA, McNulty S, Neilson RP, Ayres MP, Flannigan MD, Hanson PJ, Irland LC, Lugo AE, Peterson CJ, Simberloff D (2001) Climate change and forest disturbances: climate change can affect forests by altering the frequency, intensity, duration, and timing of fire, drought, introduced species, insect and pathogen outbreaks, hurricanes, windstorms, ice storms, or landslides. Bioscience 51:723-734

Deichmann JL, Hernández-Serna A, Delgado Cornejo JA, Campos-Cerqueira M, Aide TM (2017) Soundscape analysis and acoustic monitoring document impacts of natural gas exploration on biodiversity in a tropical forest. Ecol Ind 74:39-48

Depraetere M, Pavoine S, Jiguet F, Gasc A, Duvail S, Sueur J (2012) Monitoring animal diversity using acoustic indices: implementation in a temperate woodland. Ecol Ind 13:46-54

Digby A, Towsey M, Bell BD, Teal PD (2013) A practical comparison of manual and autonomous methods for acoustic monitoring. Methods Ecol Evol 4:675-683

Diwakar S, Balakrishnan R (2007) The assemblage of acoustically communicating crickets of a tropical evergreen forest in southern India: call diversity and diel calling patterns. Bioacoustics 16:113-135

Drewry GE, Rand AS (1983) Characteristics of an acoustic community: Puerto Rican frogs of the genus Eleutherodactylus. Copeia 4:941-953

Dumyahn SL, Pijanowski BC (2011) Soundscape conservation. Landscape Ecol 26:1327-1344

Duncan BW, Schmalzer PA (2004) Anthropogenic influences on potential fire spread in a pyrogenic ecosystem of Florida, USA. Landscape Ecol 19:153-165

Ellwood ER, Diez JM, Ibánez I, Primack RB, Kobori H, Higuchi H, Silander JA (2012) Disentangling the paradox of insect phenology: are temporal trends reflecting the response to warming? Oecologia 168:1161-1171

Engle DM, Fuhlendorf SD, Roper A, JrDM Leslie (2008) Invertebrate community response to a shifting mosaic of habitat. Rangel Ecol Manag 61:55-62

Fairbrass AJ, Rennett P, Williams C, Titheridge H, Jones KE (2017) Biases of acoustic indices measuring biodiversity in urban areas. Ecol Ind 83:169-177

Farina A, Gage SH, Salutari P (2018) Testing the ecoacoustics event detection and identification (EEDI) model on mediterranean soundscapes. Ecol Ind 85:698-715

Farina A, James P (2016) The acoustic communities: definition, description and ecological role. Biosystems 147:11-20

Farina A, Pieretti N (2014) Sonic environment and vegetation structure: a methodological approach for a soundscape analysis of a Mediterranean maqui. Ecol Inform 21:120-132

Fonseca PJ (2014) Cicada acoustic communication. In: Berthold $\mathrm{H}$ (ed) Insect hearing and acoustic communication, vol 1 . Springer, Berlin, pp 101-121
Fox J, Weisberg S (2011) An R companion to applied regression, 2nd edn. Sage, Thousand Oaks, CA

Fuller S, Axel AC, Tucker D, Gage SH (2015) Connecting soundscape to landscape: which acoustic index best describes landscape configuration? Ecol Ind 58:207-215

Gage SH, Axel AC (2014) Visualization of temporal change in soundscape power of a Michigan lake habitat over a 4-year period. Ecol Inform 21:100-109

Gasc A, Francomano D, Dunning JB, Pijanowski BC (2016) Future directions for soundscape ecology: the importance of ornithological contributions. Auk 134:215-228

Gasc A, Pavoine S, Lellouch L, Grandcolas P, Sueur J (2015) Acoustic indices for biodiversity assessments: analyses of bias based on simulated bird assemblages and recommendations for field surveys. Biol Cons 191:306-312

Gasc A, Sueur J, Jiguet F, Devictor V, Grandcolas P, Burrow C, Depraetere M, Pavoine S (2013a) Assessing biodiversity with sound: do acoustic diversity indices reflect phylogenetic and functional diversities of bird communities? Ecol Ind 25:279-287

Gasc A, Sueur J, Pavoine S, Pellens R, Grandcolas P (2013b) Biodiversity sampling using a global acoustic approach: contrasting sites with microendemics in New Caledonia. PLoS ONE 8:e65311

Guyette RP, Muzika RM, Dey DC (2002) Dynamics of an anthropogenic fire regime. Ecosystems 5:472-486

Halpin PN (1997) Global climate change and natural-area protection: management responses and research directions. Ecol Appl 7:828-843

Hannah L, Midgley G, Andelman S, Araújo M, Hughes G, Martinez-Meyer E, Pearson R, Williams P (2007) Protected area needs in a changing climate. Front Ecol Environ 5:131-138

Heinselman ML (1981) Fire intensity and frequency as factors in the distribution and structure of northern ecosystems. In: Mooney HA, Bonnicksen TM, Christensen NL, Lotan JE, Reiners WA (eds) Proceedings of the conference fire regimes and ecosystem properties, Honolulu. USDA Forest Service, General Technical Report WO-26, Washington DC, pp 7-57

Hill KB, Marshall DC, Moulds MS, Simon C (2015) Molecular phylogenetics, diversification, and systematics of Tibicen Latreille 1825 and allied cicadas of the tribe Cryptotympanini, with three new genera and emphasis on species from the USA and Canada (Hemiptera: Auchenorrhyncha: Cicadidae). Zootaxa 3985:219-251

Holm S (1979) A simple sequentially rejective multiple test procedure. Scand J Stat 6:65-70

Joo W, Gage SH, Kasten EP (2011) Analysis and interpretation of variability in soundscapes along an urban-rural gradient. Landscape Urban Plan 103:259-276

Krause B, Gage SH, Joo W (2011) Measuring and interpreting the temporal variability in the soundscape at four places in Sequoia National Park. Landscape Ecol 26:1247-1256

Kuehne LM, Padgham BL, Olden JD (2013) The soundscapes of lakes across an urbanization gradient. PLoS ONE 8:e55661

Lee BPY, Davies ZG, Struebig MJ (2017) Smoke pollution disrupted biodiversity during the 2015 El Niño fires in Southeast Asia. Environ Res Lett 12:094022

Lellouch L, Pavoine S, Jiguet F, Glotin H, Sueur J (2014) Monitoring temporal change of bird communities with 
dissimilarity acoustic indices. Methods Ecol Evol 5:495-505

Ligges U, Krey S, Mersmann O, Schnackenberg S (2013) tuneR: analysis of music. http://r-forge.r-project.org/projects/ tuner/

Lomolino MV, Pijanowski BC, Gasc A (2015) The silence of biogeography. J Biogeogr 42:1187-1196

Luczkovich JJ, Pullinger RC, Johnson SE, Sprague MW (2008) Identifying sciaenid critical spawning habitats by the use of passive acoustics. Trans Am Fish Soc 137:576-605

Méndez-Barroso LA, Vivoni ER, Watts CJ, Rodríguez JC (2009) Seasonal and interannual relations between precipitation, surface soil moisture and vegetation dynamics in the North American monsoon region. J Hydrol 377:59-70

Militino AF (2010) Mixed effects models and extensions in ecology with R. J R Stat Soc Ser A 173:938-939

Mullet TC, Gage SH, Morton JM, Huettmann F (2016) Temporal and spatial variation of a winter soundscape in southcentral Alaska. Landscape Ecol 31:1117-1137

Pausas JG, Llovet J, Rodrigo A, Vallejo R (2008) Are wildfires a disaster in the Mediterranean basin? - A review. Int J Wildl Fire 17:713-723

Pekin BK, Jung J, Villanueva-Rivera LJ, Pijanowski BC, Ahumada JA (2012) Modeling acoustic diversity using soundscape recordings and LIDAR-derived metrics of vertical forest structure in a neotropical rainforest. Landscape Ecol 27:1513-1522

Peters DCP, Lugo AE, Chapin FS III, Pickett STA, Duniway M, Rocha AV, Sawanson FJ, Laney C, Jones J (2011) Crosssystem comparisons elucidate disturbance complexities and generalities. Ecosphere 2:art81

Pieretti N, Farina A, Morri D (2011) A new methodology to infer the singing activity of an avian community: the Acoustic Complexity Index (ACI). Ecol Indic 11:868-873

Pijanowski BC, Farina A, Gage SH, Dumyahn SL, Krause BL (2011a) What is soundscape ecology? An introduction and overview of an emerging new science. Landscape Ecol 26:1213-1232

Pijanowski BC, Villanueva-Rivera LJ, Dumyahn SL, Farina A, Krause BL, Napoletano BM, Gage SH, Pieretti N (2011b) Soundscape ecology: the science of sound in the landscape. Bioscience 61:203-216

Powell BF, Schmidt CA, Halvorson WL, Anning P (2009) Vascular plant and vertebrate inventory of Chiricahua national monument (No. 2008-1023). US Geological Survey

R Core Team (2014) R: a language and environment for statistical computing. R Foundation for Statistical Computing, Vienna, Austria. http://www.R-project.org/

Röder A, Hill J, Duguy B, Alloza JA, Vallejo R (2008) Using long time series of Landsat data to monitor fire events and post-fire dynamics and identify driving factors. A case study in the Ayora region (eastern Spain). Remote Sens Environ 112:259-273

Ruse MG, Hasselquist D, Hansson B, Tarka M, Sandsten M (2016) Automated analysis of song structure in complex birdsongs. Anim Behav 112:39-51

Sanborn AF, Phillips PK (2013) Biogeography of the cicadas (Hemiptera: Cicadidae) of North America, north of Mexico. Diversity 5:166-239
Sankupellay M, Towsey M, Truskinger A, Roe P (2015) Visual fingerprints of the acoustic environment. In: Proceedings of the IEEE international symposium on big data visual analytics. IEEE, New York

Schafer RM (1977) The Soundscape. Our sonic environment and the tuning of the world. Destiny Books, Vancouver

Stephens SL, Agee JK, Fule PZ, North MP, Romme WH, Swetnam TW, Turner MG (2013) Managing forests and fire in changing climates. Science 342:41-42

Sueur J, Aubin T, Simonis C (2008a) Seewave: a free modular tool for sound analysis and synthesis. Bioacoustics $18: 213-226$

Sueur J, Farina A, Gasc A, Pieretti N, Pavoine S (2014) Acoustic indices for biodiversity assessment and landscape investigation. Acta Acust United Acust 100:772-781

Sueur J, Pavoine S, Hamerlynck O, Duvail S (2008b) Rapid acoustic survey for biodiversity appraisal. PLoS ONE 3:e4065

Swetnam TW (1993) Fire history and climate change in giant sequoia groves. Science 262:885

Swetnam TW, Baisan CH, Caprio AC, Brown PM (1992) Fire history in a Mexican oak-pine woodland and adjacent montane conifer gallery forest in southeastern Arizona. Paper presented at the symposium on the ecology and management of Oak and Associated Woodlands: perspectives in the Southwestern United States and Northern Mexico, Sierra Vista, AZ, USA, April 27-30, 1992

Syphard AD, Clarke KC, Franklin J (2007) Simulating fire frequency and urban growth in southern California coastal shrublands, USA. Landscape Ecol 22:431-445

Towsey M, Wimmer J, Williamson I, Roe P (2014a) The use of acoustic indices to determine avian species richness in audio-recordings of the environment. Ecol Inform 21:110-119

Towsey M, Zhang L, Cottman-Fields M, Wimmer J, Zhang J, Roe P (2014b) Visualization of long-duration acoustic recordings of the environment. Proc Comput Sci 29:703-712

Truskinger A, Cottman-Fields M, Johnson D, Roe P (2013) Rapid scanning of spectrograms for efficient identification of bioacoustic events in big data. Paper presented at IEEE 9th international conference on eScience (eScience), Beijing, China, 22-25 October, 2013

Turner MG (2010) Disturbance and landscape dynamics in a changing world. Ecology 91:2833-2849

Turner MG, Hargrove WW, Gardner RH, Romme WH (1994) Effects of fire on landscape heterogeneity in Yellowstone National Park, Wyoming. J Veg Sci 5:731-742

Turner MG, Romme WH, Gardner RH, O'Neill RV, Kratz TK (1993) A revised concept of landscape equilibrium: disturbance and stability on scaled landscapes. Landscape Ecol 8:213-227

U.S. Geological Survey, Gap Analysis Program (GAP) (2011) National land cover, Version 2

U.S. Geological Survey and the U.S. Forest Service (2013) Monitoring trends in burn severity assessment of fire information: AZ3182010921120110508. Raster digital data for satellite imagery and derived data for fire perimeters. Sioux Falls, South Dakota or Salt Lake City, Utah. http://edc.usgs.gov or http://www.fs.fed.us/eng/rsac/ and http://www.mtbs.gov/ 
Vankat JL (1977) Fire and man in Sequoia National Park. Ann Assoc Am Geogr 67:17-27

Venables WN, Ripley BD (2002) Modern applied statistics with S, 4th edn. Springer, New York

Viedma O, Melia J, Segarra D, Garcia-Haro J (1997) Modeling rates of ecosystem recovery after fires by using Landsat TM data. Remote Sens Environ 61:383-398

Villanueva-Rivera LJ, Pijanowski BC (2012) Pumilio: a webbased management system for ecological recordings. Bull Ecol Soc Am 93:71-81

Villanueva-Rivera LJ, Pijanowski BC (2015) soundecology: soundscape ecology. $\mathrm{R}$ package version 1.3.1. http:// CRAN.R-project.org/package=soundecology

Warshall P (1995) The Madrean sky island archipelago: a planetary overview. In: DeBano LF, Gottfried GJ, Hamre RH, Edminster CB, Dolliott PFF, Ortega-Rubio A (eds) Biodiversity and management of the Madrean Archipelago: the sky islands of Southern United States and Northwestern Mexico. USDA Forest Service General Technical Report RM-GTR-264, pp 6-18

Watts CJ, Scott RL, Garatuza-Payan J, Rodriguez JC, Prueger JH, Kustas WP, Douglas M (2007) Changes in vegetation condition and surface fluxes during NAME 2004. J Clim 20:1810-1820

White PS, Pickett STA (1985) Natural disturbance and patch dynamics: an introduction. In: Pickett STA, White PS (eds) The ecology of natural disturbance and patch dynamics. Academic Press, New york, pp 3-13

Wildlife Acoustics, Inc. Bioacoustics software and field recording equipment website. http://www. wildlifeacoustics.com/. Accessed 1 January 2014

Williams KS, Smith KG, Stephen FM (1993) Emergence of 13-yr periodical cicadas (Cicadidae: Magicicada): phenology, mortality, and predator satiation. Ecology 74:1143-1152

Yeates GW, Lee WG (1997) Burning in a New Zealand snowtussock grassland: effects on vegetation and soil fauna. N Zeal J Ecol 1:73-79

Zhao Z, Zhang SH, Xu ZY, Bellisario K, Dai NH, Omrani H, Pijanowski BC (2017) Automated bird acoustic event detection and robust species classification. Ecol Inform 39:99-108 
Soundscapes reveal disturbance impacts: biophonic response to wildfire in the Sonoran Desert Sky Islands

Amandine Gasc*, Benjamin Gottesman, Dante Francomano, Jinha Jung, Mark Durham, Jason Mateljak and Bryan C. Pijanowski

*(Corresponding Author). e-mail: amandine.gasc@gmail.com

Online Resource 1: Site replicate selection process 
To reduce sources of variability due to differences between sites within each ecological system, we chose sites that met the following conditions: i) Independence: sites of the same system were never chosen in the same patch, and all sites of the same system were separated from each other by at least $200 \mathrm{~m}$. Each site was located in a patch represented by at least 2 pixels $(1$ pixel $=30$ * $30 \mathrm{~m})$. ii) Accessibility: the sites were all relatively accessible, meaning that they were located between 20 and $400 \mathrm{~m}$ from a road and that the difference in elevation between the sites and the closest point on the road was less than $100 \mathrm{~m}$. iii) Comparability: sites within each ecological system were chosen to be as similar as possible in elevation, aspect, slope, and phenology (based on data collected before the Horseshoe 2 Burn.) Aspect, elevation, and slope were calculated from the topographic data compiled in the Arizona statewide Digital Elevation Model (1-arc second DEM; USGS 3D Elevation Program), and phenological similarity between locations was calculated from phenology data based on the Normalized Difference Vegetation Index (NDVI) of the area before the fire (USDA Forest Services ForWarn tool).

A total of 132 locations with suitable accessibility were selected (one location $=1$ pixel of $30 * 30$ $\mathrm{m})$. From this list of possible locations, we determined independence and comparability of the locations by a distance matrix using Pavoine et al.'s generalization of Gower's distance (Pavoine et al. 2009) considering the burn severity, the ecological system, the slope, the elevation, the aspect and the phenology. Because four parameters were used to define phenology, each of these parameters were weighted by 0.25 (see below for detail concerning these parameters and calculations). For each system, a hierarchical cluster analysis based on the calculation of the Ward's minimum variance was performed on the distances between the locations. The results were represented by a dendrogram using the function 'hclust' $\{$ stats $\}$ in R (R Core Team 2014). 
A final selection of 24 sites was made in the field, using the dendrograms of the sites, the maps of land cover (Fry et al. 2011; US Geological Survey 2011) and burn information (U.S. Geological Survey and the U.S. Forest Service 2013), and the necessary help of Chiricahua National Monument staff members (Figure 1; for the exact coordinates of the sites, see Table S1). Software used for this site selection were ArcMap 10.1, Excel 2013, and Rx64 3.0.2.

\section{Phenological similarity calculation}

ForWarn is an Early Warning System (EWS) tool for detection and tracking of regionally evident forest change (Spruce et al. 2012). It provides continuous phenological information over the entire United States of America. We received the ForWarn data over the study area from the USGS ForWarn group. These data contain phenological information since 2000. In this study, we used phenological information over a 10-year time period prior to the wildfire disturbance (2000 2010).

To characterize these 10 years of phenological information, we i) split the entire 10 -year phenology time-series into 10 curves to obtain one time-series per year, ii) modeled each phenology with a Gaussian function, and iii) used the four parameters $(a, b, c$, and $d)$ to describe the Gaussian curve, where $a$ is the estimated maximum value of the amplitudes, $b$ is the estimated mean value of the distribution, $c$ is the estimated standard deviation value of the distribution and $d$ is the estimated error. In phenological models, $a$ can be interpreted as the maximum greenness occurring over the year, $b$ can be interpreted as the period of the year associated with that maximum of greenness occurrence, and $c$ will indicate how long the greenness lasted in the year. To fit a Gaussian function over the 10 separated phenological curves, we used the Levenberg-Marquardt (LM) algorithm (Moré 1978). Since the LM algorithm 
goes through an iterative process to estimate parameters of the Gaussian function, we needed to have initial estimates of these parameters. We used a maximum value location as an initial estimate of the mean, a maximum amplitude value as an initial estimate of the amplitude, and a fixed value ( 1 in this case) as an initial estimate of the standard deviation. This was implemented using Python and the SciPy library (Jones et al. 2001).

\section{References}

Fry J, Xian G, Jin S, Dewitz J, Homer C, Yang L, Barnes C, Herold N, Wickham J (2011) Completion of the 2006 National Land Cover Database for the Conterminous United States. PE\&RS 77:858-864.

Jones E, Oliphant E, Peterson P, et al. (2001) SciPy: Open Source Scientific Tools for Python. http://www.scipy.org/ [Online; accessed 2018-02-02].

Moré JJ (1978) The Levenberg-Marquardt algorithm: implementation and theory. In Numerical analysis, pp. 105-116. Springer, Berlin Heidelberg.

Pavoine S, Vallet J, Dufour AB, Gachet S, Daniel H (2009) On the challenge of treating various types of variables: application for improving the measurement of functional diversity. Oikos 118:391-402.

Spruce JP, Hargrove W, Gasser G, Smoot J, Kuper PD (2012) Contribution of national near real time MODIS forest maximum percentage NDVI change products to the US ForWarn system.

U.S. Geological Survey, Gap Analysis Program (GAP). May 2011. National Land Cover, Version 2. Available: http://edc.usgs.gov 
U.S. Geological Survey and the U.S. Forest Service (2013). Monitoring Trends in Burn Severity assessment of Fire Information: AZ3182010921120110508. Raster digital data for satellite imagery and derived data for fire perimeters. Sioux Falls, South Dakota or Salt Lake City, Utah. Available: http://edc.usgs.gov or http://www.fs.fed.us/eng/rsac/ and http://www.mtbs.gov/. 
Soundscapes reveal disturbance impacts: biophonic response to wildfire in the Sonoran Desert Sky Islands

Amandine Gasc*, Benjamin Gottesman, Dante Francomano, Jinha Jung, Mark Durham, Jason Mateljak and Bryan C. Pijanowski

*(Corresponding Author). e-mail: amandine.gasc@gmail.com

Online Resource 3: Table S1. Coordinates and conditions of the 24 acoustically monitored sites. In the site name the first two characters refer to the ecological system, the third and fourth refer to the burn severity level, and the two last characters indicated the replicate number. "Madrean pinyon-juniper woodland" and "Madrean pine-oak forest and woodland" have been reduced to respectively read "Madrean pinyon-juniper" and "Madrean pine-oak" in the table. 


\begin{tabular}{|c|c|c|c|c|}
\hline Site Name & Ecological system & Burn severity & Latitude & Longitude \\
\hline H1-NO-R1 & Madrean pinyon-juniper & non-burned & 32.00317 & -109.36307 \\
\hline H1-NO-R2 & Madrean pinyon-juniper & non-burned & 32.00148 & -109.36005 \\
\hline H1-NO-R3 & Madrean pinyon-juniper & non-burned & 32.02108 & -109.35809 \\
\hline H1-HI-R1 & Madrean pinyon-juniper & burned (high) & 32.02728 & -109.32102 \\
\hline H1-HI-R2 & Madrean pinyon-juniper & burned (high) & 32.00623 & -109.30917 \\
\hline H1-HI-R3 & Madrean pinyon-juniper & burned (high) & 32.02929 & -109.34772 \\
\hline H2-NO-R1 & Madrean encinal & non-burned & 32.01234 & -109.31639 \\
\hline H2-NO-R2 & Madrean encinal & non-burned & 32.01376 & -109.31838 \\
\hline H2-NO-R3 & Madrean encinal & non-burned & 32.00825 & -109.36015 \\
\hline H2-HI-R1 & Madrean encinal & burned (high) & 32.02692 & -109.32002 \\
\hline H2-HI-R2 & Madrean encinal & burned (high) & 32.02808 & -109.34794 \\
\hline H2-HI-R3 & Madrean encinal & burned (high) & 32.02565 & -109.36239 \\
\hline H3-NO-R1 & Mongollon chaparral & non-burned & 32.01153 & -109.31603 \\
\hline H3-NO-R2 & Mongollon chaparral & non-burned & 32.00829 & -109.31944 \\
\hline H3-NO-R3 & Mongollon chaparral & non-burned & 32.00778 & -109.32167 \\
\hline H3-ME-R1 & Mongollon chaparral & burned (medium) & 32.00964 & -109.31686 \\
\hline H3-ME-R2 & Mongollon chaparral & burned (medium) & 32.00737 & -109.31545 \\
\hline H3-ME-R3 & Mongollon chaparral & burned (medium) & 32.00687 & -109.36070 \\
\hline H4-NO-R1 & Madrean pine-oak & non-burned & 32.02190 & -109.33536 \\
\hline H4-NO-R2 & Madrean pine-oak & non-burned & 32.02401 & -109.35757 \\
\hline H4-NO-R3 & Madrean pine-oak & non-burned & 32.02180 & -109.3293 \\
\hline H4-HI-R1 & Madrean pine-oak & burned (high) & 32.02117 & -109.34047 \\
\hline H4-HI-R2 & Madrean pine-oak & burned (high) & 32.02271 & -109.34473 \\
\hline H4-HI-R3 & Madrean pine-oak & burned (high) & 32.01361 & -109.3278 \\
\hline
\end{tabular}


Soundscapes reveal disturbance impacts: biophonic response to wildfire in the Sonoran Desert Sky Islands

Amandine Gasc*, Benjamin Gottesman, Dante Francomano, Jinha Jung, Mark Durham, Jason Mateljak and Bryan C. Pijanowski

*(Corresponding Author). e-mail: amandine.gasc@gmail.com

Online Resource 2: Sonic Timelapse Builder Description 
Sonic Timelapse Builder (STLB) is a MAX/MSP program that creates sonic timelapses (STLs) from multiple audio files.

STLB reads a set of files specified by the user. These are sorted sequentially by filename and then loaded into the program. STLB then determines a playback sequence, playing back one section or slice from each file. The length and density of the final sequence is defined by the controls "slice length" and "overlap amount" (Figure S2). "Slice length" defines how long the section from each file runs for, while "overlap amount" defines how many slices are playing concurrently at any one time.

In effect, the playback section of STLB is a macro-scale granular process; it creates a synchronous audio stream comprising many audio slices (Roads 1991). On playback, the program distributes the playback sequence data for each slice to one of the four stereo players, cycling this distribution sequentially through them. Each player also shapes the slice with an amplitude envelope to avoid clicks. The envelope shape used is a pure Gaussian curve to attempt to create a smooth fade between slices. The stereo output of the four players is summed together, and then this output is rendered as a single stereo file recorded to a disk.

To use this program, please visit https://github.rcac.purdue.edu/PijanowskiGroup/Sonic-Timelapse-Builder.

\section{References}

Roads C (1991) Asynchronous granular synthesis. Representation of musical signals. MIT Press, pp 143-186. 

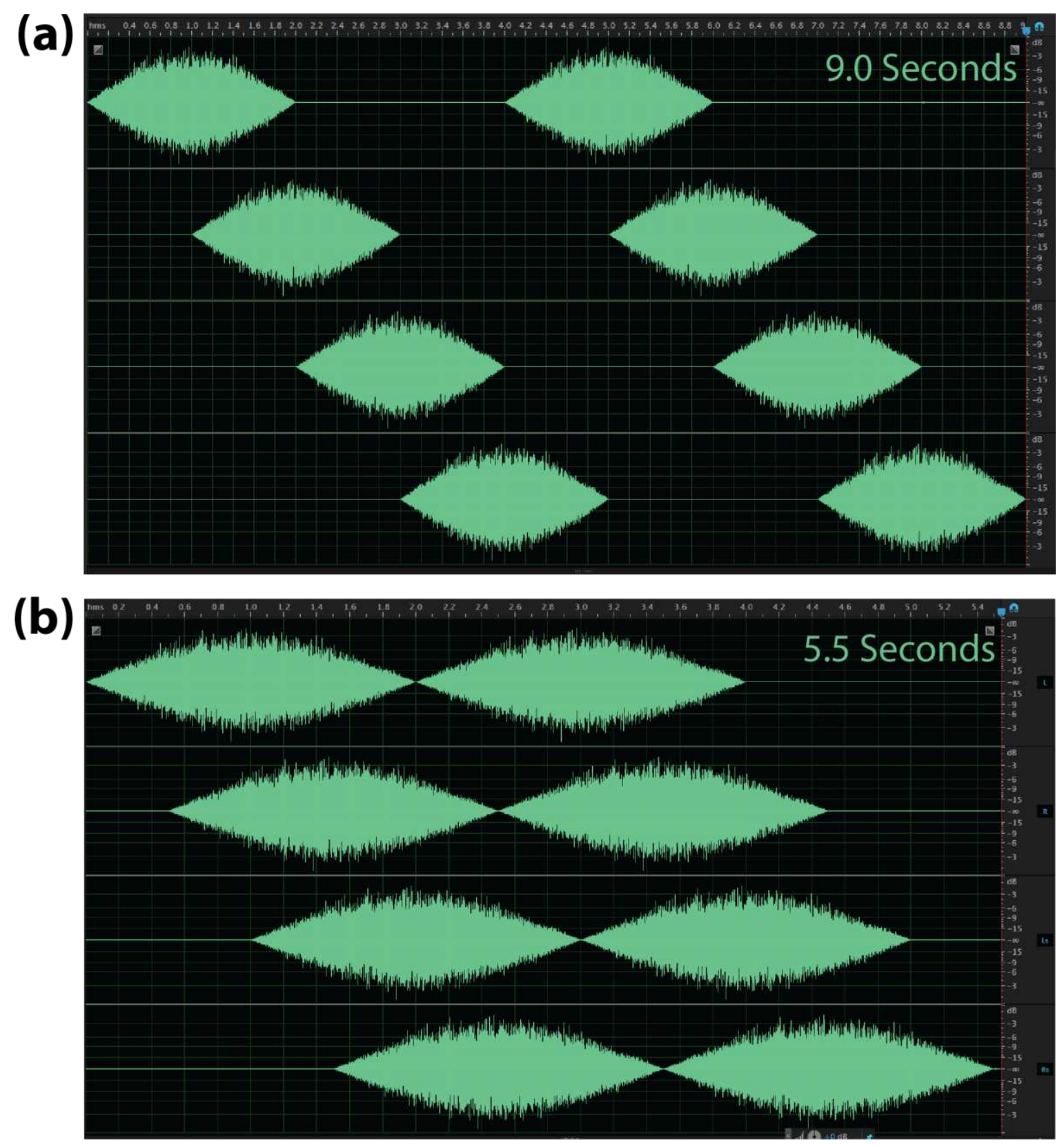

Figure S2: Sonic Timelapse Builder overlap visualization. Both (a) and (b) display timelapses formed from eight 2000-ms samples of white noise that pass through gaussian-shaped envelopes. (a) Overlap 1, where sequential files begin 50\% into the preceding file, and (b) Overlap 2 where sequential files begins $25 \%$ into the preceding file. 
Soundscapes reveal disturbance impacts: biophonic response to wildfire in the Sonoran Desert Sky Islands

Amandine Gasc*, Benjamin Gottesman, Dante Francomano, Jinha Jung, Mark Durham, Jason Mateljak and Bryan C. Pijanowski

*(Corresponding Author). e-mail: amandine.gasc@gmail.com

Online Resource 5: Figure S1. Diel acoustic variation for October in non-burned and burned sites. 

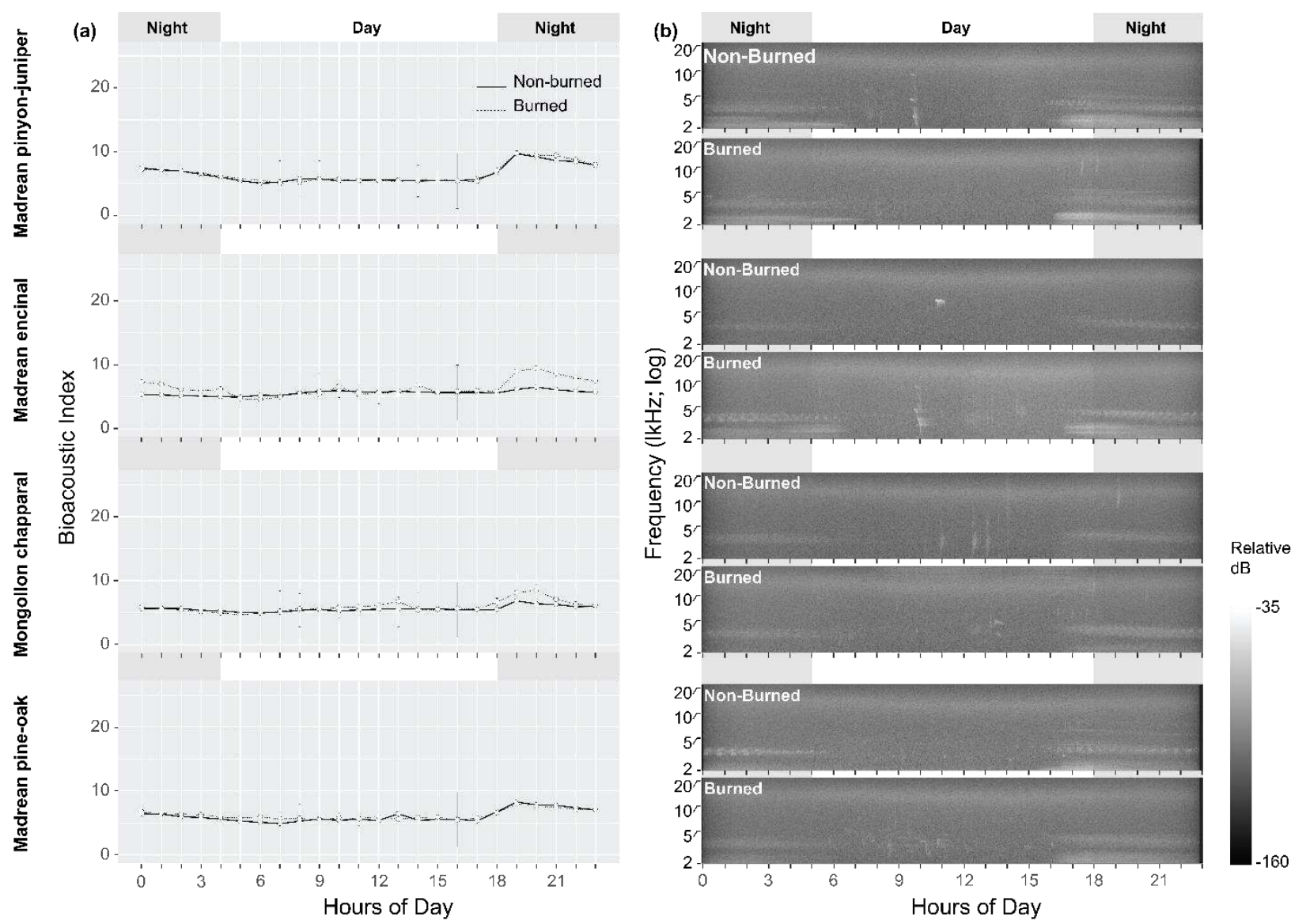

Figure S1: Diel acoustic variation for October in non-burned and burned sites. (a) Variation of the Bioacoustic Index for each of the four ecological systems. Each point represents the average and each bar represents the standard error associated with the index value. The number of sites used for averages varied due to missing data. Sites that were averaged include: 2 non-burned and 1 burned site for Madrean pinyon-juniper, 2 non-burned and 1 burned sites for Madrean encinal, 3 non-burned and 3 burned sites for Mogollon chaparral, and 2 non-burned and 3 burned sites for Madrean pine-oak. (b) Spectrograms of the corresponding sonic timelapses (STLs) for nonburned and burned areas of each system. Spectrograms were derived from 13.7 sec STLs with a high pass filter set at $2 \mathrm{kHz}$. 
Soundscapes reveal disturbance impacts: biophonic response to wildfire in the Sonoran Desert Sky Islands

Amandine Gasc*, Benjamin Gottesman, Dante Francomano, Jinha Jung, Mark Durham, Jason Mateljak and Bryan C. Pijanowski

*(Corresponding Author). e-mail: amandine.gasc@gmail.com

Online Resource 4: Tables of the linear mixed model results and associated assumption validation summary. 
Table S2: Linear mixed model results and assumption validation summary for daytime of the month of June. The p-values have been corrected following the Bonferroni method. L refers to Lambda coefficient, $\mathrm{P}$ to $\mathrm{P}$-value and $\mathrm{N}$ to the number of values (number of recordings analyzed). The dependent variable ( $\mathrm{Y})$ has been modify by $\mathrm{Y}^{\wedge} \mathrm{L}$.

\begin{tabular}{|c|c|c|c|c|c|c|}
\hline Hour of day & $\mathbf{P}$ & $\mathbf{N}$ & $\mathbf{L}$ & Normality & Homoscedasticity & LinearityCollinearity \\
\hline 5 & 1 & 306 & -2 & validated & validated & validated validated \\
\hline 6 & 0.15 & 304 & -2 & validated & validated & validated validated \\
\hline 7 & 1 & 301 & -2 & Not validated & validated & validated validated \\
\hline 8 & 0.045 & 284 & -1.7 & Not validated & validated & validated validated \\
\hline 9 & $1.04 \mathrm{e}-07 * * *$ & 242 & -1.1 & validated & validated & validated validated \\
\hline 10 & 0.0045 & 197 & -1.9 & Not validated & validated & validated validated \\
\hline 11 & 0.14 & 111 & -2 & Not validated & validated & validated validated \\
\hline 12 & 0.02 & 86 & -2 & Not validated & validated & validated validated \\
\hline 13 & 1 & 66 & -2 & Not validated & validated & validated validated \\
\hline 14 & 0.48 & 18 & -2 & Not validated & validated & validated validated \\
\hline 15 & 1 & 21 & -2 & validated & validated & validated validated \\
\hline 16 & 0.48 & 21 & -2 & Not validated & validated & validated validated \\
\hline 17 & 1 & 37 & -2 & Not validated & validated & validated validated \\
\hline
\end{tabular}


Table S3: Linear mixed model results and assumption validation summary for nighttime of the month of October. The p-values have been corrected following the Bonferroni method. L refers to Lambda coefficient, $\mathrm{P}$ to $\mathrm{P}$-value and $\mathrm{N}$ to the number of values (number of recordings analyzed). The dependent variable (Y) has been modify by $\mathrm{Y}^{\wedge} \mathrm{L}$.

\begin{tabular}{|c|c|c|c|c|c|c|c|}
\hline Hour of day & $\mathbf{P}$ & $\mathbf{N}$ & $\mathbf{L}$ & Normality & Homoscedasticity & Linearity & Collinearity \\
\hline 18 & 1 & 173 & 0 & not validated & not validated & not validated & not validated \\
\hline 19 & 0.18 & 206 & -1 & not validated & validated & not validated & validated \\
\hline 20 & 0.12 & 206 & -1.4 & not validated & validated & not validated & validated \\
\hline 21 & 0.23 & 230 & -1.3 & not validated & validated & not validated & validated \\
\hline 22 & 0.85 & 230 & -0.9 & validated & validated & validated & validated \\
\hline 23 & 0.85 & 236 & -1.1 & validated & validated & not validated & validated \\
\hline 0 & 0.37 & 259 & 0.8 & not validated & not validated & not validated & validated \\
\hline 1 & 0.85 & 236 & -0.9 & validated & validated & validated & validated \\
\hline 2 & 0.85 & 233 & -0.6 & validated & validated & validated & validated \\
\hline 3 & 0.85 & 223 & -0.4 & validated & validated & validated & validated \\
\hline 4 & 0.85 & 227 & -0.7 & validated & validated & validated & validated \\
\hline
\end{tabular}


Soundscapes reveal disturbance impacts: biophonic response to wildfire in the Sonoran Desert Sky Islands

Amandine Gasc*, Benjamin Gottesman, Dante Francomano, Jinha Jung, Mark Durham, Jason Mateljak and Bryan C. Pijanowski

*(Corresponding Author). e-mail: amandine.gasc@gmail.com

Online Resource 6: Description of the Timelapses files 
Table S4: Description of the Timelapses files added as 16 Online Resources.

\begin{tabular}{|c|c|c|c|c|}
\hline File name & Ecological system & Fire & Month & High pass filter \\
\hline ESM_7.mp2 & Madrean pinyon-juniper & not-burned & June & $2 \mathrm{kHz}$ \\
\hline ESM_8.mp2 & Madrean pinyon-juniper & burned & June & $2 \mathrm{kHz}$ \\
\hline ESM_9.mp2 & Madrean pinyon-juniper & not-burned & October & $2 \mathrm{kHz}$ \\
\hline ESM_10.mp2 & Madrean pinyon-juniper & burned & October & $2 \mathrm{kHz}$ \\
\hline ESM_11.mp2 & Madrean encinal & not-burned & June & $2 \mathrm{kHz}$ \\
\hline ESM_12.mp2 & Madrean encinal & burned & June & $2 \mathrm{kHz}$ \\
\hline ESM_13.mp2 & Madrean encinal & not-burned & October & $2 \mathrm{kHz}$ \\
\hline ESM_14.mp2 & Madrean encinal & burned & October & $2 \mathrm{kHz}$ \\
\hline ESM_15.mp2 & Mongollon chapparal & not-burned & June & $2 \mathrm{kHz}$ \\
\hline ESM_16.mp2 & Mongollon chapparal & burned & June & $2 \mathrm{kHz}$ \\
\hline ESM_17.mp2 & Mongollon chapparal & not-burned & October & $2 \mathrm{kHz}$ \\
\hline ESM_18.mp2 & Mongollon chapparal & burned & October & $2 \mathrm{kHz}$ \\
\hline ESM_19.mp2 & Madrean pine-oak & not-burned & June & $2 \mathrm{kHz}$ \\
\hline ESM_20.mp2 & Madrean pine-oak & burned & June & $2 \mathrm{kHz}$ \\
\hline ESM_21.mp2 & Madrean pine-oak & not-burned & October & $2 \mathrm{kHz}$ \\
\hline ESM_22.mp2 & Madrean pine-oak & burned & October & $2 \mathrm{kHz}$ \\
\hline
\end{tabular}

